\title{
Design of control system of Smart Home based on embedded Linux
}

\author{
Yang $\mathrm{Li}^{1}$, and Yunliang Wang ${ }^{2}$, \\ ${ }^{1}$ School of Electrical Engineering of Tianjin University of Technology, China \\ ${ }^{2}$ Tianjin Key Laboratory for Control Theory \& Application in Complicated System, Tianjin University \\ of Technology, China
}

Keywords: Linux; embedded; Smart Home; Qt

\begin{abstract}
Based on embedded Linux system, the paper puts forward a kind of remote control system for household appliances. The system mainly uses the way of combination of WiFi and GPRS, which are wireless connection technology, and TCP protocol as the primary communication protocol to realize information interaction, and finally control household appliances by the method of the infrared decoding and encoding. Through the mobile client connect to the Internet, and then connect to the server, each subsystem can be controlled. The system realized controlling most household appliances.
\end{abstract}

\section{Introduction}

Currently, embedded smart home system has become a popular choice for the development of the smart home. Smart Home is a residential platform. It uses integrated wiring, network communication technology, automatic control technology, audio and video technology to integrate household-related facilities, builds a management system of efficient residential facilities and home affairs agenda improves home safety, convenience, comfort, artistry, and creates an eco-friendly living environment. The most important thing of smart home products is practical and considers it as the core. The products abandon those functions which are not useful and merely used of decorations. Their main features are product availability, ease of using and user-friendly based. In the daily home life, several of forms of intelligent teminal is essential such as: intelligent remote control, mobile touch screen computers, cell phones, PDA and so on. And it is important to control the system and get the needing information at any time. With the rapid development of smart phones this year, smart phones are more and more chose as the operator terminal by smart home products.

\section{The system structure of Smart Home}

The Smart home used ARM11 development board as the total server, and used the Linux2.6.28 operating system. Qt/Embedded graphical management interface run on the controller, the user can manage all kinds of home appliances through the touch screen. The GUI of the server response the user' s operations through the background process to achieve the goal of managing the electrical appliances. The remote controller chose the Android mobile phone, realized the connection to the server through TCP protocol programming, sent the operation information to the server by operating the mobile phone operation interface, according to the information received, the background program managed the electrical appliances.

According to the products' classification of Smart Home system, the interface management mainly achieve the following functions: security system, home appliance control system, intelligent lighting system, home audio system and system settings module. Figure 1 is a simple assumption diagram of the system. 


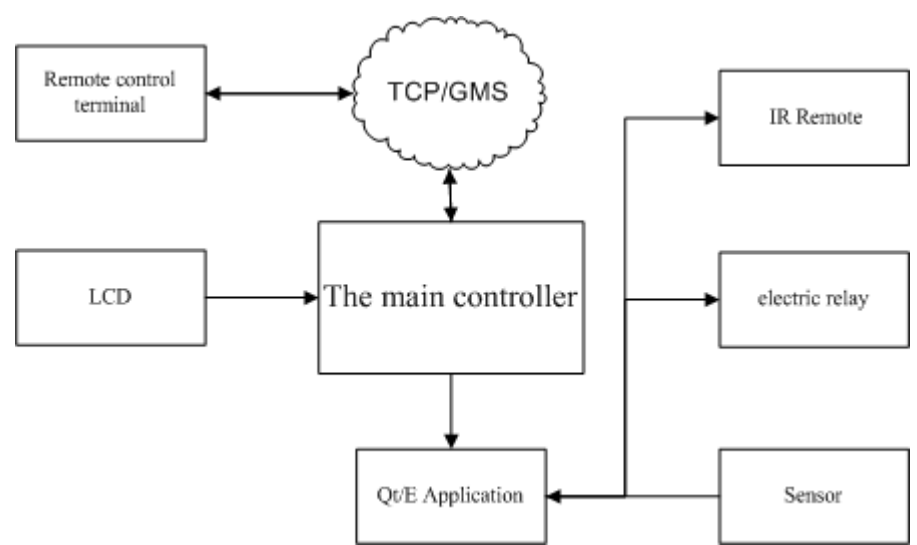

Fig.1 system structure diagram

\section{System Hardware Design}

In this system, the control end and the controlled end is the daily necessities, witch are not controlled directly by the system. So it is needed to design the devices that can control the controlled end directly, and these devices do not need very strong handling capacity, only need to receive instructions from the server and impose corresponding penalties, so the 89C52 Series MCU is chose as the processor. The Wi-Fi module chose RMO4 module, the server's microprocessor of system used the S3C6410 microprocessor of Samsung Corp, witch is is a high performance embedded processor, support many LCD and many I/O port. The server also integrates various peripheral interfaces, such as Ethernet, USB, serial port and so on, witch can completely meet the requirements of Smart Home. Fig. 2 is the hardware block diagrams of the system.

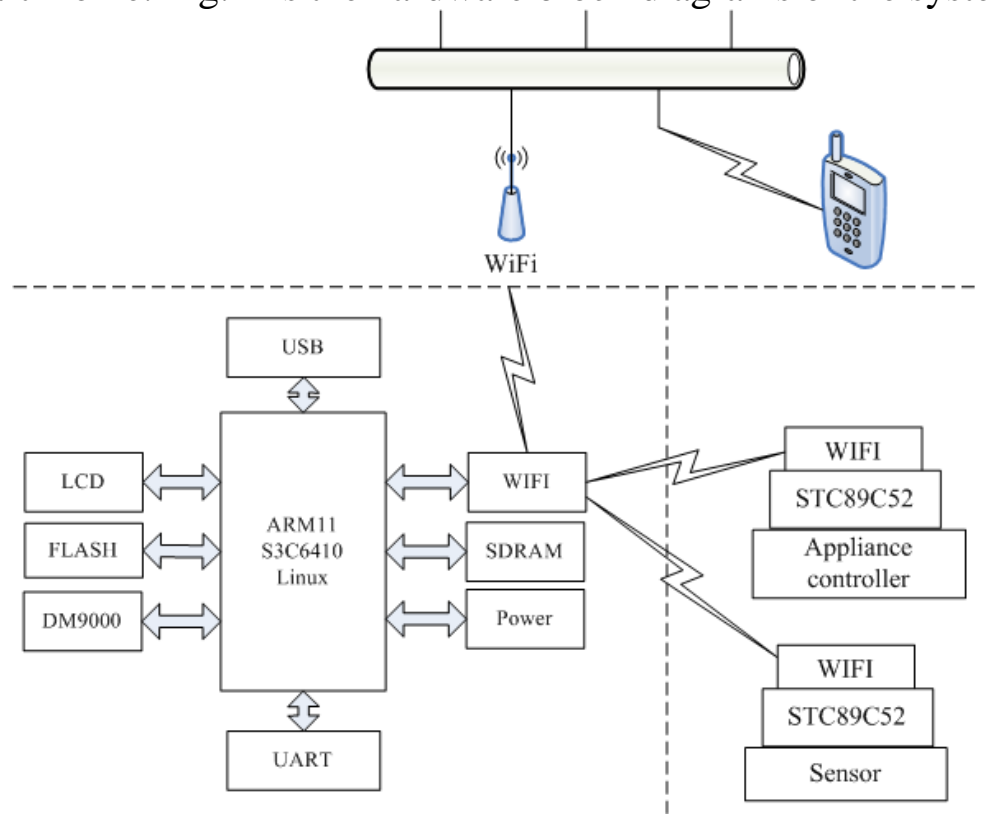

Fig. 2 hardware block diagram

\section{System Software Design}

The system software mainly includes the transplantation of Linux operating system, Qt graphical interface application program, Qt for Android interface application program, 89C52 microcontroller program etc.. The operating system adopts Linux2.6.28 system.

Embedded Linux system. Embedded Linux is an open-source embedded system based on Linux system, oLinux has the rich software resources, portability and good stability, support for multi-threaded, multi-user, multi process.

Qt graphical user interface application program. Because of supporting multi-platform, portability, Qt has been widely used in embedded Linux system. The core mechanism of Qt is the 
mechanism of signal and slot that is a powerful , object-oriented communication mechanism, it is more flexible than the callback mechanism in general. The relationship between the signals and slots can be one to one, many to one and one to many.

The Qt graphical user interface application run on the server, its main function is to act as a TCP server, receive and process the information from client, send information to client. In addition, Qt graphical interface program also deal with the user's operating instructions. Fig. 4 is the application's flow chart.

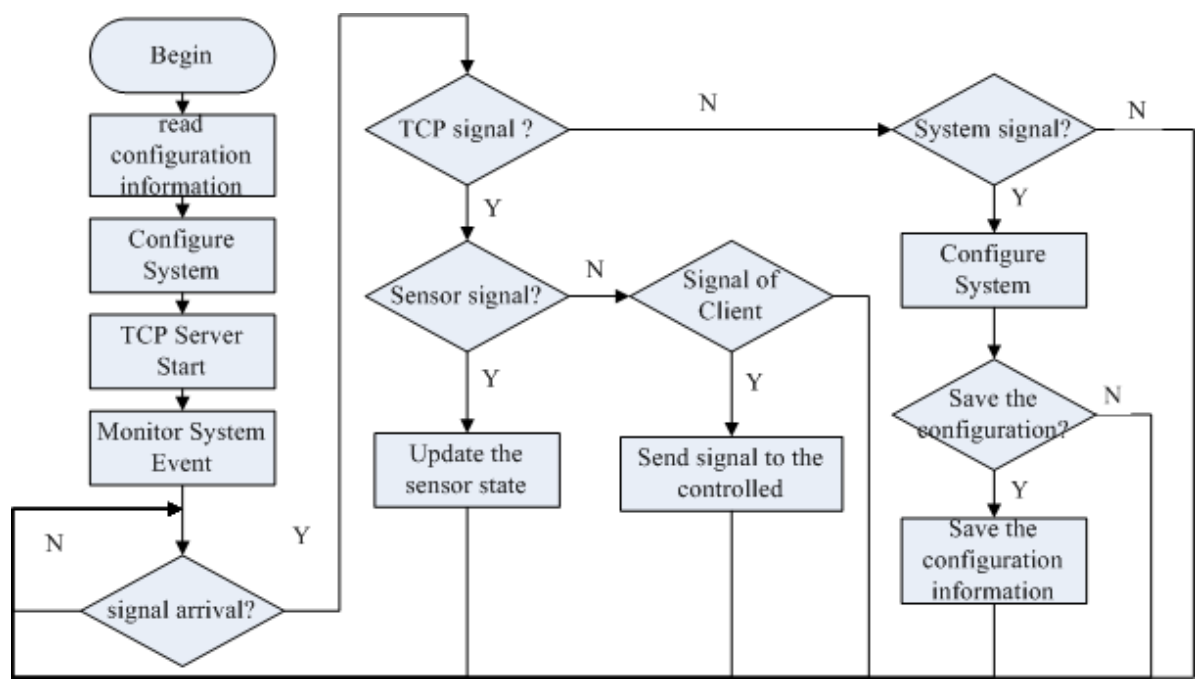

Fig. 3 the flow chart of server's application

Interface application program of Qt for Android. Qt for Android is a project that makes Qt support to develop Android applications.Qt began to support the Android platform formally from version fifth, support the Android API of level 10 or above.

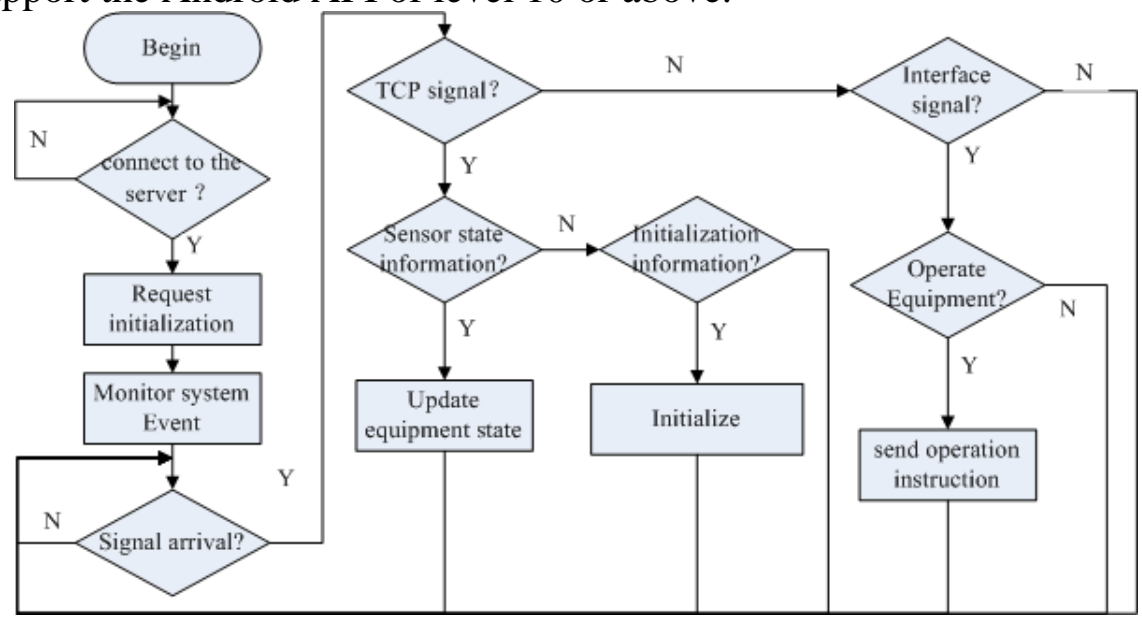

Fig. 4 the flow chart of the android client application

Qt provides a QAndroidJni-Object class to realize code interaction between Qt and Android. In this class callObjectMethod function and callStaticMethod function are most important.Before using this class, the Android function needed completed, for example, realizing sending message must state the permission (android.permission.SEND_SMS), than he callObjectMethod function or the callStaticMethod function can be used to call the Java class interface to send text messages.

The application of Qt for android acts as the operating interface of the remote controller witch is a android mobile phone, its main function is to communicate with the server and respond the user's operations.Fig. 4 is the flow chart of the android client application.

He system is stable and reliable in work, Fig. 5 is the server's interface control interface, and Fig.6 is the mobile phone's control interface, click the icon to enter through the interface room room equipment list interface, click the list of devices can realize the control of household electric appliances. 


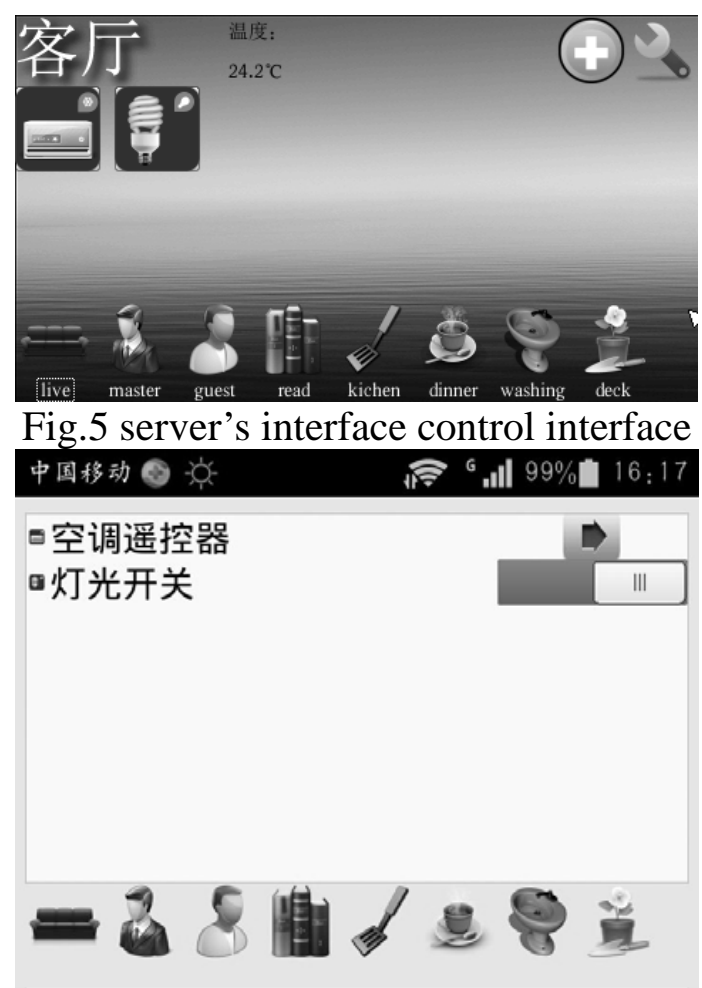

Fig.6 mobile phone’s control interface

\section{Conclusions}

This paper design a Smart Home Control System based on the embedded Linux system, witch introduce the system architecture, the hardware design and the software design in detail.Embedded Linux terminal interface and android client interface was completed based on Qt’s portability.

\section{Reference}

[1]Xiu Yang,Yang Jing.Smart Home system based on Android[J],Technological Pioneers, 2014,09:57.

[2]Yu Wen-jun, Ling Zhi-hao.Research on a smart Home System Based on Internet of Things[J].Process Automation Instrumentation,2011,08:56-59.

[3]Guo Wen-tao, He Yi-gang.Research and Design of Smart Home Remote Monitoring System[J].Computer Measurement \& Control,2011,09:2109-2112.

[4]Chun-yue Bi, Yun-peng Liu, Ren-fang Wang. Research of key technologies for embedded Linux based on ARM. Computer Application and System Modeling (ICCASM), 2010 International Conference on,22-24 Oct. 2010.

[5]He Xiang,Meng Xiao-hua.Design and implementation of human-computer interface in embedded intelligent home system[J].Computer Engineering and Design,2010,10:2166-2168.

[6]Chen Jing, Xiang Wei.QT/Embedded Transplantation and Application Based on Embedded Linux[J].Computer and Information Technology,2013,01:57-59. 